\begin{tabular}{|c|l|}
\hline Title & Selenium-Ioaded zeolite (ZSM-5): II. Photoinduced phenomena \\
\hline Author(s) & Saitoh, A kira; Tanaka, Keiji \\
\hline Citation & $\begin{array}{l}\text { Journal of A pplied Physics, 105(11), 113531 } \\
\text { https://doi.org/10.1063/1.3143612 }\end{array}$ \\
\hline Issue Date & 2009-06-01 \\
\hline Doc URL & http://hdl.handle.net/2115/38779 \\
\hline Rights & $\begin{array}{l}\text { Copyright 2009A A merican Institute of Physics. This article may be downloaded for personal use only. A ny other use } \\
\text { requires prior permission of the author and the A merican Institute of Physics. }\end{array}$ \\
\hline Type & article \\
\hline File Information & 105-11_113531.pdf \\
\hline
\end{tabular}

Instructions for use 


\title{
Selenium-loaded zeolite (ZSM-5): II. Photoinduced phenomena
}

\author{
Akira Saitoh and Keiji Tanaka ${ }^{a)}$ \\ Department of Applied Physics, Graduate School of Engineering, Hokkaido University, Sapporo 060-8628, \\ Japan
}

(Received 19 March 2009; accepted 4 May 2009; published online 11 June 2009)

\begin{abstract}
Photoinduced phenomena in Se-loaded ZSM-5 single crystals have been studied through electron-spin resonance, optical absorption, and photoluminescence spectroscopy. Marked exposure effects appear at room temperature. Upon illumination of visible light, an electron-spin signal at $g \approx 2.00$ increases by an order, subgap absorption of $10^{3} \mathrm{~cm}^{-1}$ appears, and photoluminescence at subgap regions weakens to a half. Timescales of these photoinduced changes including recoveries are not the same, which suggests different origins for the three changes. The spin increase evinces bond scission in single Se chains. (C) 2009 American Institute of Physics. [DOI: 10.1063/1.3143612]
\end{abstract}

\section{INTRODUCTION}

Photoinduced phenomena in chalcogenide glasses have attracted considerable interest for a long time, while underlying mechanisms remain speculative. ${ }^{1-3}$ To examine the mechanism, we may choose the simplest material, amorphous Se $(a-\mathrm{Se})$, which exhibits three kinds of photoinduced phenomena: photocrystallization at $\sim 350 \mathrm{~K},{ }^{2,4,5}$ photodarkening and related changes at $100-300 \mathrm{~K}^{6-8}$ and photoinduced electron-spin resonance $\left(\right.$ ESR) signals at $\sim 10 \mathrm{~K}^{9-11}$ However, the disordered structure consisting of entangled chains (and rings) held together by van der Waals forces ${ }^{12-14}$ still makes the elucidation of photoelectroatomic processes difficult.

To understand the principal role of electronic excitation in Se, we may explore photoinduced phenomena in single clusters. Nagaya et al. ${ }^{15}$ demonstrated that free $\mathrm{Se}_{5}{ }^{+}$(odd number) ions in vacuum are unstable under illumination, while the details have not been reported. On the other hand, considerable studies have been performed for Se clusters loaded into zeolites, which are transparent and porous $\mathrm{Si}-$ Al-O crystals with a variety of kinds. ${ }^{16}$ Reported phenomena for Se are photoinduced absorption in $\mathrm{Na} A,{ }^{17}$ photostructural changes in mordenite ${ }^{18}$ and zeolite $4 \mathrm{~A},{ }^{19}$ photoinduced fragmentation in zeolite $Y$ (Ref. 20) and $A,{ }^{21}$ and photoionization in cancrinite. ${ }^{22}$

However, these studies are limited in experimental conditions. First, some studies employ powderlike zeolites, ${ }^{18,19}$ and accordingly, Se atoms deposited onto zeolite surfaces may affect or govern observed properties. Second, all the zeolites previously employed are fairy ionic $(\mathrm{Al} / \mathrm{Si} \geq 0.2),{ }^{17-22}$ which inevitably causes strong interaction between $\mathrm{Se}$ and inner-wall atoms of the pore in zeolite, as theoretically predicted. ${ }^{23,24}$ Third, some researchers employed zeolites having substantially larger pores than atomic diameters of chalcogen, ${ }^{17-22}$ which may mask single molecular properties. Finally, previous studies detected photoinduced effects at low temperatures, ${ }^{17-19}$ except a few on photofragmentation and ionization at room temperature. ${ }^{20-22}$

\footnotetext{
a) Author to whom correspondence should be addressed. Electronic mail: keiji@eng.hokudai.ac.jp.
}

Therefore, it is interesting to examine if Se clusters loaded into a zeolite having covalent and tight pores present some photoinduced phenomena.

We here investigate the phenomenon in Se-loaded ZSM-5 $(z-\mathrm{Se})$. This zeolite is fairly covalent $(\mathrm{Al} / \mathrm{Si}=0.02)$ and has pores with free and framework diameters of $\sim 0.56$ and $\sim 0.83 \mathrm{~nm}^{25,26}$ The authors have suggested in a previous paper $^{27}$ that in this zeolite, Se takes single fourfold (not threefold) helices with an external diameter of $\sim 0.68 \mathrm{~nm}$. The Se content in ZSM-5 crystals is estimated at $\sim 10^{15}$ / piece and ESR measurements give a spin density of $\sim 10^{13}$ / piece, which can be ascribed to oxygen chargetransfer complexes. Taking these results into account, we envisage that the Se chain has lengths of $\sim 10^{2}$ atoms on average, both ends being terminated by the oxygen complexes. In addition, Raman-scattering spectra suggest the existence of $\mathrm{Se}_{2}$, which is known to be stable in Se vapor. ${ }^{28}$ [Whether the chain length varies at (i) $2-10^{2}$ or (ii) 2 and $\sim 10^{2}$ is not known.] The optical absorption edge is located at $\sim 2.2 \mathrm{eV}$, while the absorption edge is substantially gradual, which implies varied chain lengths and/or defective structures.

The present work demonstrates, for the first time, that $\mathrm{Se}$ in ZSM-5 exhibits three kinds of photoinduced phenomena at room temperature. Photoinduced ESR evinces that single Se chains are cut under illumination at room temperature, which was predicted by computer simulations, ${ }^{29,30}$ while no direct evidence has been obtained so far. Photoinduced optical absorption and photoluminescence (PL) fatigue also appear, the origins being discussed. These results will add new insight into the photoinduced phenomenon in chalcogenide glasses. ${ }^{1-3}$

\section{EXPERIMENTS}

Sample preparation was described in Ref. 27. In brief, Se chunks with $6 \mathrm{~N}$ purity were vaporized at $300{ }^{\circ} \mathrm{C}$ and impregnated into ZSM-5 crystals held at $\sim 150{ }^{\circ} \mathrm{C}$ in vacuum. Photoinduced phenomena, under illumination of laser light with photon energies of 2.0-2.6 eV and intensities of 1-40 $\mathrm{mW}$, were inspected in three properties. First, ESR spectra were obtained using an $X$-band continuous-wave spectrometer at $290 \mathrm{~K}$. Concentration of unpaired electron spins was 

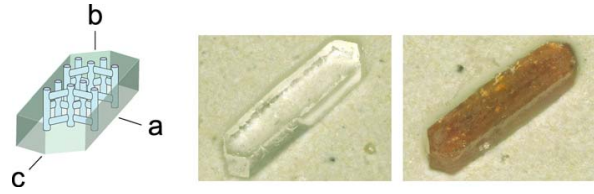

FIG. 1. (Color online) An illustration of ZSM-5 with crystal axes and magnified pores and photographs of ZSM-5 and a Se-loaded ZSM-5.

calibrated using $\mathrm{CuSO}_{4} \cdot 5 \mathrm{H}_{2} \mathrm{O}$ as a standard. Second, photoinduced absorption was investigated using a conventional spectrometer. Induced absorption was monitored within $\sim 5$ min after interrupting light excitation. Third, PL was measured using several lasers and a triple-polychromator system at room temperature. Spectral shapes were calibrated using a black-body radiation.

\section{RESULTS}

Figure 1 shows a schematic view of ZSM-5 with photographs of the zeolite and a $z$-Se sample employed in the present experiments. The zeolite has a typical size of 40, 40, and $200 \mu \mathrm{m}$ for the $a, b$, and $c$ directions. As described in Ref. 27, the transparent ZSM-5 changes to brownish orange after Se loading.

Figure 2 shows ESR spectra obtained for a pile ( $\sim 1000$ pieces) of $z$-Se. As reported in Ref. 27, pristine $z$-Se gives a sharp signal (a), suggesting spins with a density per zeolite crystal of $\sim 10^{13} /$ piece, which is ascribed to Seoxygen charge-transfer complexes. ${ }^{31}$ Katayama et al. ${ }^{18}$ reported a similar sharp signal for Se in mordenite, while the origin is not specified. As shown in Fig. 2(b), the ESR signal is photoenhanced. Illumination of $2.0 \mathrm{eV}$ light with an intensity of $\sim 1 \mathrm{~mW}\left(\sim 100 \mathrm{~mW} / \mathrm{cm}^{2}\right)$ for a few minutes at room temperature has increased the signal with the spectral shape unchanging. The increase is an order, suggesting $\sim 10^{14} \mathrm{spin} /$ piece, which scarcely changes upon prolonged illumination. Quantum efficiency of this photoinduced process, estimated from a ratio of the spin density $\left(\sim 10^{14} \mathrm{spin} /\right.$ piece) divided by the absorbed photon $\operatorname{dose}^{32}\left(10^{15}\right.$ $-10^{16} /$ piece), is $10^{-2}-10^{-1}$. The increased signal stays stable after terminating the illumination, at least, for 3 days. It is mentioned that exposures using a $2.3 \mathrm{eV}$ laser and a Xe lamp have brought similar increases in the spin signal, which suggest that the saturated spin density does not depend critically

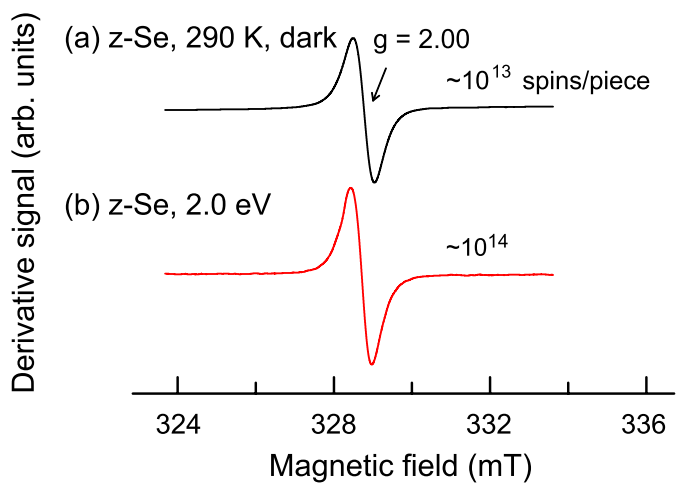

FIG. 2. (Color online) ESR spectra of $z$-Se at room temperature (a) in the dark and (b) after illumination of light with $2.0 \mathrm{eV}, \sim 1 \mathrm{~mW}$, and $\sim 5 \mathrm{~min}$.

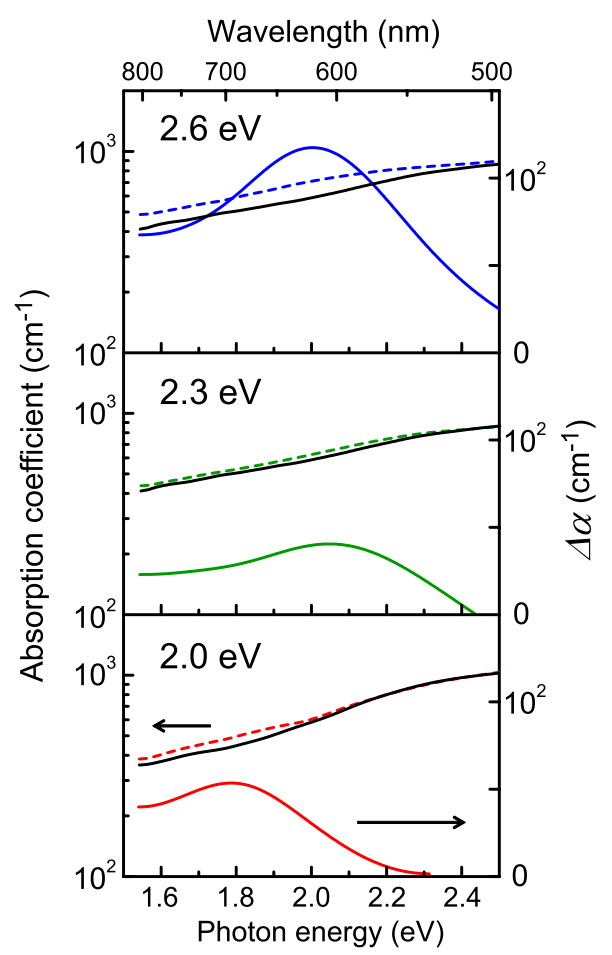

FIG. 3. (Color online) Photoinduced absorption changes (after saturation) by laser lines $(2.0-2.6 \mathrm{eV})$ in $z$-Se.

on the photon energy of excitation. It should be mentioned that the present ESR result is contrastive to those previously reported. ${ }^{9-11,18}$ For instance, $a$-Se does not give dark ESR signals, and illumination of bandgap light at cryogenic temperatures induces broad signals $(\sim 20 \mathrm{mT})$ at $g \sim 2$ with spin densities of $10^{16}-10^{19} \mathrm{~cm}^{-3}$, which is ascribed to dangling bonds of $\mathrm{Se}^{1}$

Figure 3 shows that $z$-Se undergoes photoinduced absorption at $1.6-2.4 \mathrm{eV}$ at room temperature. In detail, the induced absorption has a peak, which is located at $\sim 1.8 \mathrm{eV}$ under excitation of $2.0 \mathrm{eV}$ and at $\sim 2.0 \mathrm{eV}$ under excitations of 2.3 and $2.6 \mathrm{eV}$. It should be noted that the growing timescale of the induced absorption is much longer than that of the photoinduced ESR. For instance, under the $2.0 \mathrm{eV}$ excitation, the absorption saturation occurs after $\sim 1 \mathrm{~h}$ (Fig. 4), being longer by an order than that of the spin increase. This

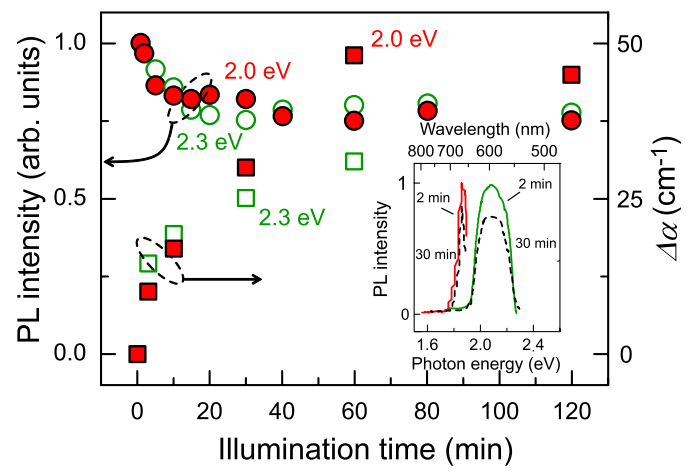

FIG. 4. (Color online) PL fatigue (circles) and induced absorption (squares) and induced by laser light of $2.0 \mathrm{eV}$ and $1 \mathrm{~mW}$ (solid red) and $2.3 \mathrm{eV}$ and 40 $\mathrm{mW}$ (open green). Inset shows PL spectra after exposures of $2 \mathrm{~min}$ (minimal detection period, colored solid lines) and $30 \mathrm{~min}$ (dashed lines) with $2.0 \mathrm{eV}$ (left, red) and $2.3 \mathrm{eV}$ (right, green) light. 
induced absorption is stable, at least, for $\sim 10 \mathrm{~h}$. It is mentioned that intense excitation of $2.8 \mathrm{eV}$ has gradually bleached the sample, and ultimately the sample becomes transparent (not shown), which may be due to sublimation of Se.

As reported elsewhere, ${ }^{32}$ upon excitation of laser light with photon energies of 2.0-2.8 eV, $z$-Se presents broad PL in visible spectral regions, $1.8-2.6 \mathrm{eV}$, at room temperature. This visible PL becomes stronger by an order at $80 \mathrm{~K}$. It has been confirmed using another polychromator system that no PL appears at near infrared regions $(\lambda \leq 2 \mu \mathrm{m})$.

The PL fatigues upon light excitation at room temperature. For instance, as shown in Fig. 4, upon irradiation of the $2.0 \mathrm{eV}$ light, PL weakens to $\sim 70 \%$ with a timescale of $\sim 1 \mathrm{~h}$, which is similar to that of the induced absorption. The fatigue level is, for excitation of $2.0-2.5 \mathrm{eV}, 50 \%-70 \%$, which seem to vary from sample to sample. However, in contrast to those in the ESR and absorption changes, the PL fatigue has recovered at room temperature with a timescale of $\sim 10 \mathrm{~h}$. Then, the PL again weakens under repeated excitation.

To the authors' knowledge, the present work gives the first demonstration of the photoinduced absorption and PL fatigue in Se. It should be also mentioned here that Ramanscattering spectroscopy could detect no structural modifications corresponding to the three photoinduced changes.

\section{DISCUSSION}

We have discovered, for $z$-Se at room temperature, the photoinduced changes in ESR, optical absorption, and PL. Here, the first problem is if these three changes arise from a unique structural change. The answer seems negative because the related timescales of photoinductions and thermal recoveries are different. As mentioned, upon illumination of $2.0 \mathrm{eV}$ light of $\sim 100 \mathrm{~mW} / \mathrm{cm}^{2}$, the spin increase, induced optical absorption, and PL fatigue have occurred, respectively, with the timescales of $\sim 5 \mathrm{~min}, \sim 1 \mathrm{~h}$, and $\sim 1 \mathrm{~h}$. Moreover, only the PL fatigue has recovered after storage in the dark at room temperature for $\sim 10 \mathrm{~h}$. $(2.3 \mathrm{eV}$ light has given similar features, while those may be affected by lightpenetration effects.). Therefore, we should consider the origins of these photoinduced changes one-by-one, taking the suggested Se structure ${ }^{27}$ into account.

Mechanism of the photoenhanced ESR relies upon an interpretation of the dark signal because of the very similar spectral shapes. Abkowitz ${ }^{31}$ and others ${ }^{33}$ ascribed the sharp ESR signal in solid Se to the oxygen charge-transfer complex, which may be denoted as $-\mathrm{Se}^{+} \mathrm{O}_{2}^{-}$, where -Se symbolizes a chain end. This interpretation provides a model for the photoinduced ESR in $z$-Se that light illumination cuts single Se chains, a kind of chain fragmentation process, and the broken ends immediately form the oxygen complexes as $-\mathrm{Se}^{+} \mathrm{O}_{2}{ }^{-} \mathrm{O}_{2}{ }^{-} \mathrm{Se}^{+}-$. It seems that $\mathrm{O}_{2}$ exists anywhere since it can easily penetrate into the pore due to the small elliptical shape of $\sim 0.3$ and $\sim 0.4 \mathrm{~nm}$. Otherwise, $\mathrm{O}_{2}$ may be supplied from the inner walls of ZSM-5 pores. Taking the Se density of $\sim 10^{15} /$ piece (Ref. 27) and the photoenhanced spin density of $\sim 10^{14}$ / piece, we now assume that the chain length is reduced to $\sim 10$ atoms on average. Similar photofragmentations seem to occur in Se clusters in other zeolites. ${ }^{22,28}$

The present observation has provided a direct evidence of photoinduced chain scission at room temperature. ${ }^{34}$ It is known that in solid and molecular Se, the valence band and HOMO level are made from lone-pair electron states, not from bonding states. ${ }^{23,24,35}$ Hence, if excitation of a lone-pair electron can cut a covalent bond is not clear. However, the photoinduced ESR evinces the scission, consistent with a molecular-dynamics simulation for an infinitely long $\mathrm{Se}$ chain at $500 \mathrm{~K},{ }^{30}$ which demonstrates photoinduced stateshybridization leading to bond breaking. It is plausible that such bond scission assists the photoinduced crystallization. $^{2,4,5}$

However, for the present interpretation, some problems may be posed. For instance, why does not the chain shortening accompany a blueshift of the HOMO-LUMO gap, as expected from a simple quantum-well calculation? A plausible reason is that the mean-free path of electronic carriers is still shorter than or comparable to the chain length of $\sim 10$ atoms so that the effect cannot appear. Otherwise, the shift arising from the quantum-well effect is estimated at $\sim 0.1 \mathrm{eV}$, which could not be experimentally resolved due to limited shapes of the present sample and the gradual absorption edge. We may also ask why the ultimate chain length is $\sim 10$ atoms. A speculation is that the length just corresponds to the mean-free path (or thermalization distance) of excited carriers, and the chain end works as an efficient nonradiative recombination center, which suppresses further bond breaking.

For the induced absorption, the origin is ambiguous. The spectral shape suggests that the induced absorption is not the photodarkening, ${ }^{6-8}$ which denotes a nearly parallel redshift of the absorption edge. ${ }^{1-3}$ The photodarkening seems to arise from enhanced disordering in interchain interaction, ${ }^{1}$ and accordingly, its nonexistence in single Se chains is reasonable. On the other hand, it cannot be known if the induced absorption can be regarded as the midgap absorption extending at $E_{g} / 2 \sim E_{g}\left(E_{g} \text { is the optical gap }\right)^{1}$ since the lower energy side $\left(\sim E_{g} / 2\right)$ could not be inspected due to the limited size of $z$-Se samples. Since the induced absorption shows a longer growing timescale than that of the ESR, we should assume some ESR inactive structural changes, which occur in the shortened Se chains. For instance, the chain may be photooxidized as $-\mathrm{Se}-\mathrm{O}-\mathrm{Se}-$ or $-\mathrm{Se}-\mathrm{O}_{2}-\mathrm{Se}-$. It should be mentioned that photoinduced absorptions in previous studies are demonstrated for Se in powdered zeolites, ${ }^{17,18}$ which may be influenced by Se deposited onto zeolite surfaces.

For an interpretation of the PL fatigue at room temperature, the origin of the PL should be known first. Here, we underline the small Stokes shift of $0.1-0.2 \mathrm{eV}$, which implies that the PL arises from exciton (electron-hole pair) recombination. Since the exciton is produced in the deformable single chains, polaronic excitons may be responsible for the PL. This view seems to be consistent with a theoretical result by Ikawa and Fukutome ${ }^{23}$ and previous experimental results for $t$-Se (Ref. 36) and for Se in zeolite. ${ }^{37}$ It seems that Se in ZSM-5 works as a dye in a wide-gap host. It should be noted that the small Stokes shift in $z$-Se is contrastive to those 
reported for $a$-Se (Ref. 38), $t$-Se (Ref. 39), and Se-cancrinite, ${ }^{22}$ in which the PL peak-energy $E_{\mathrm{PL}}$ satisfies the so-called half-gap rule $E_{\mathrm{PL}} \simeq E_{g} / 2$, which is widely observed in chalcogenide glasses and corresponding crystals, ${ }^{38}$ although the origin remains controversial. ${ }^{1,40}$

How can we interpret the PL fatigue in $z$-Se? Since it occurs with a similar timescale to the induced absorption, while it thermally recovers at room temperature, we may envisage additional changes to that causing the induced absorption. For instance, ESR inactive biexcitons are selftrapped at the distorted segment or the oxidized sites as $-\mathrm{Se}^{+}-\mathrm{O}_{2}(\mathrm{O})^{2-}-\mathrm{Se}^{+}-$. Provided that such excitons have a lifetime of $\sim 10 \mathrm{~h}$, the PL may fatigue due to a limited density of the trapping sites.

\section{CONCLUSIONS}

It has been demonstrated that Se-loaded ZSM-5 exhibits at room temperature photoinduced phenomena including photoinduced ESR, subgap absorption, and PL fatigue. The photoinduced ESR gives a direct evidence of the bond scission in single Se chains. The subgap absorption and PL fatigue occur with longer timescales than that of ESR, and only the PL shows a recovery. Structural changes giving rise to the induced absorption are largely speculative. PL possibly arises from self-trapped (polaronic) excitons in single $\mathrm{Se}$ chains, and the fatigue can be related to a finite exciton lifetime.

\section{ACKNOWLEDGMENTS}

The authors would like to thank Dr. Y. Kiyozumi for supplying ZSM-5 samples, Dr. T. Hiraoki for ESR measurement, Dr. A. V. Kolobov and Dr. V. V. Poborchii for unpublished information, Dr. N. Terakado for PL measurement, and graduate students S. Okamoto and K. Noda for experimental assistance. The authors also acknowledge a financial support from Nippon Sheet Glass Foundation for Material Science and Engineering.

${ }^{1}$ K. Shimakawa, A. Kolobov, and S. R. Elliott, Adv. Phys. 44, 475 (1995) ${ }^{2}$ M. A. Popescu, Non-Crystalline Chalcogenides (Kluwer, Dordrecht, 2000)

${ }^{3}$ Photo-Induced Metastability in Amorphous Semiconductors, edited by A V. Kolobov (Wiley-VCH, Weinheim, 2003).

${ }^{4}$ J. Dresner and G. B. Stringfellow, J. Phys. Chem. Solids 29, 303 (1968)

${ }^{5}$ K. Ishida and K. Tanaka, Phys. Rev. B 56, 206 (1997).
${ }^{6}$ K. Tanaka and A. Odajima, Solid State Commun. 43, 961 (1982).

${ }^{7}$ A. Reznik, B. J. M. Lui, J. A. Rowlands, S. D. Baranovskii, O. Rubel, V. Lyubin, M. Klebanov, and S. O. Kasap, J. Appl. Phys. 100, 113506 (2006).

${ }^{8}$ A. Reznik, M. Klebanov, and V. Lyubin, J. Appl. Phys. 105, 013518 (2009).

${ }^{9}$ S. G. Bishop, U. Strom, and P. C. Taylor, Phys. Rev. B 15, 2278 (1977).

${ }^{10}$ A. V. Kolobov, M. Kondo, H. Oyanagi, A. Matsuda, and K. Tanaka, Phys. Rev. B 58, 12004 (1998).

${ }^{11}$ L. N. Blinov, Glass Phys. Chem. 29, 203 (2003).

${ }^{12}$ P. Andonov, J. Non-Cryst. Solids 47, 297 (1982).

${ }^{13}$ B. W. Corb, W. D. Wei, and B. L. Averbach, J. Non-Cryst. Solids 53, 29 (1982).

${ }^{14}$ K. Nakamura and A. Ikawa, Phys. Rev. B 67, 104203 (2003).

${ }^{15}$ K. Nagaya, T. Hayakawa, M. Yao, and H. Endo, J. Non-Cryst. Solids 205-207, 807 (1996).

${ }^{16}$ http://www.iza-structure.org/databases/

${ }^{17}$ V. N. Bogomolov, V. V. Poborchii, S. V. Kholodkevich, and S. I. Shagin, JETP Lett. 38, 532 (1983).

${ }^{18}$ Y. Katayama, M. Yao, Y. Ajiro, M. Inui, and H. Endo, J. Phys. Soc. Jpn. 58, 1811 (1989).

${ }^{19}$ K. Maruyama, T. Tsuzuki, M. Yao, and H. Endo, Surf. Rev. Lett. 3, 711 (1996).

${ }^{20}$ A. Goldbach, M. Grimsditch, L. Iton, and M. L. Saboungi, J. Phys. Chem. B 101, 330 (1997).

${ }^{21}$ V. V. Poborchii, Prog. Zeolite and Microporous Mater. 105, 631 (1997).

${ }^{22}$ V. V. Poborchii, G. G. Lindner, and M. Sato, J. Chem. Phys. 116, 2609 (2002).

${ }^{23}$ A. Ikawa and H. Fukutome, J. Phys. Soc. Jpn. 59, 1002 (1990).

${ }^{24}$ A. A. Demkov and O. F. Sankey, J. Phys.: Condens. Matter 13, 10433 (2001).

${ }^{25}$ G. T. Kokotailo, S. L. Lawton, D. H. Olson, and W. M. Meier, Nature (London) 272, 437 (1978).

${ }^{26}$ H. van Koningsveld, J. C. Jansen, and H. van Bekkum, Zeolites 10, 235 (1990).

${ }^{27}$ A. Saitoh and K. Tanaka, J. Appl. Phys. 105, 113530 (2009).

${ }^{28}$ A. Goldbach, L. Iton, M. Grimsditch, and M. L. Saboungi, J. Am. Chem. Soc. 118, 2004 (1996).

${ }^{29}$ A. Ikawa and H. Fukutome, J. Phys. Soc. Jpn. 60, 3067 (1991).

${ }^{30}$ K. Hoshino, F. Shimojo, and T. Nishida, J. Phys. Soc. Jpn. 68, 1907 (1999).

${ }^{31}$ M. Abkowitz, J. Chem. Phys. 46, 4537 (1967).

${ }^{32}$ A. Saitoh and K. Tanaka, Solid State Commun. 149, 750 (2009).

${ }^{33}$ Selenium, edited by R. A. Zingaro and W. C. Cooper (Van Nostrand Reinhold, New York, 1974).

${ }^{34}$ In the present work, no experiments have been performed for nonoxygenized $z$-Se, and accordingly, whether the bond scission gives the Se dangling bonds is not known.

${ }^{35}$ M. Kastner, Phys. Rev. Lett. 28, 355 (1972).

${ }^{36}$ S. Tutihasi and I. Chen, Phys. Rev. 158, 623 (1967).

${ }^{37}$ L. He, Z. X. Shen, G. Gu, L. Qin, and S. H. Tang, Chem. Phys. Lett. 300, 504 (1999).

${ }^{38}$ R. A. Street, Adv. Phys. 25, 397 (1976).

${ }^{39}$ H. Lundt and G. Weiser, Solid State Commun. 48, 827 (1983).

${ }^{40}$ J. Ristein, P. C. Taylor, W. D. Ohlsen, and G. Weiser, Phys. Rev. B 42, 11845 (1990). 